\title{
COMMENTARY
}

\section{Peri-operative hemodynamic therapy: only large clinical trials can resolve our uncertainty}

\author{
Neil MacDonald and Rupert M Pearse* \\ See related research by Cecconi et al., http://ccforum.com/content/15/3/R132
}

\begin{abstract}
In this issue of Critical Care, Cecconi and colleagues report the findings of a small trial of goal-directed hemodynamic therapy (GDT) in patients undergoing major orthopedic surgery under regional anesthesia. This is an interesting trial and the first of which we are aware to test the efficacy of GDT in this patient group. The findings suggest that this intervention is associated with improved adverse event rates after surgery. However, in this trial, as in all small trials of GDT, the potential for bias leaves some uncertainty regarding how widely the findings should be implemented. Such limitations may be impossible to completely eliminate from trials of complex interventions, but large multicenter trials may allow us to substantially decrease bias and improve the generalizability of the findings.
\end{abstract}

The peri-operative use of fluid and inotropes guided by cardiac output monitoring is variously known as optimization, flow-guided therapy, and goal-directed therapy (GDT). In this issue of Critical Care, Cecconi and colleagues [1], who are experienced researchers in this field, report the findings of an interesting single-center trial of GDT in patients undergoing hip replacement under regional anaesthesia. The findings of this investigation suggest that GDT may be associated with reduced adverse event rates after orthopedic surgery. There is a clear need to improve survival for patients undergoing major surgery $[2,3]$, and in many small trials, GDT has been associated with better clinical outcomes, particularly following high-risk procedures [4-6]. This beneficial effect appears to be related to improvements in

${ }^{*}$ Correspondence: rupert.pearse@bartsandthelondon.nhs.uk Intensive care Research Office, Barts and The London School of Medicine and Dentistry, Queen Mary's University of London, 4th Floor Holland Wing, Royal London Hospital, Whitechapel High Street, London E1 1BB, UK microvascular flow and tissue oxygenation [7]. The findings of previous studies have suggested benefit in patients undergoing proximal femoral fracture repair $[8,9]$, but we are unaware of any previous trials of GDT in elective orthopedic surgery. Consequently, there has been little evidence to inform the use of GDT in this large population of patients. There are distinct challenges associated with the design and conduct of GDT trials, and these challenges are not easily solved. Thus, the findings of this and all previous GDT trials must be interpreted in the context of the design choices that the investigators have made.

GDT is a term that describes a potentially more effective and objective method to determine the optimal dose of intravenous fluid and inotropic therapy through the use of a clinical algorithm. Ordinarily, the clinician will administer fluid or inotropic therapy (or both) on a subjective basis according to their preferred clinical endpoints. Hence, there is significant variability in clinical practice which, in a small trial, must be replaced by a control group algorithm designed to reflect best usual care. Failure to use this approach will expose the investigators to the possible accusation of manipulating the trial outcome through poor control group care. In the recent past, even the suggestion of such impropriety has proven damaging for highly respected investigators [10]. In the trial of Cecconi and colleagues [1], the choice of arterial pressure as a control group end-point for fluid therapy illustrates the dilemma. Fluid challenges are commonly administered to correct hypotension. However, under regional anaesthesia, arterial hypotension is more likely to result from vasodilatation than hypovolemia. We could therefore argue that usual practice is incorrect in this case and yet more effective control group care will diminish any apparent treatment effect of GDT. It is easy to criticize control group care in clinical trials of complex interventions though much harder to find a better alternative. In large multicenter trials, the variable nature of usual care is much less likely to lead to an erroneous finding. Even then, it would be advisable to recommend some treatment standards to avoid practice misalignment. Less critically, the GDT algorithm must result in 
adequate intervention in the majority of patients while minimizing potential harm. In the trial of Cecconi and colleagues, only $65 \%$ of patients achieved the predetermined target for oxygen delivery $\left(\mathrm{DO}_{2}\right)$. This observation likely reflects a GDT algorithm that incorporates judicious limits to the dose of inotropic therapy. It seems clear that the continued pursuit of hemodynamic goals in patients who do not respond is harmful [11]. The adverse effects of fluid and inotropic therapy will be most serious for patients with significant heart disease. In this respect, it would have been helpful to report the timing of cardiovascular complications. Arrhythmias may be triggered by hypovolemia or myocardial ischemia, but the effect of study group allocation would have a more transient effect on this category of complications than for others such as infection.

Blinding is another important potential source of bias in GDT trials. GDT is a complex intervention that involves human decision making. In this context, it is impossible to fully blind investigators to study group allocation. Small GDT trials are particularly vulnerable to bias, and investigators may find it difficult to demonstrate the adequacy of their procedures for assessing nonbinary outcomes such as complications. This problem can be minimized through the use of blinded assessors who determine clinical outcomes according to predetermined criteria. In the report by Cecconi and colleagues, these processes could be better described to clearly demonstrate methodological rigor. The use of scoring systems such as the postoperative morbidity survey (POMS) may prove useful for assessment of minor morbidity, but once again, large multicenter trials would appear to be the most effective way to reduce bias.

This interesting trial adds another piece to the jigsaw of optimal peri-operative hemodynamic therapy. The apparent efficacy of this approach during elective orthopedic surgery in self-ventilating patients under regional anesthesia is of particular interest. We agree with the authors that GDT has a potential role in the treatment of such patients. We also agree that further research is required to confirm their findings. Small clinical trials add to the literature, but the lack of confirmation in large trials is a significant limitation of the evidence base for GDT. We hope that this interesting investigation will be followed by a robustly designed multicenter trial designed to confirm or refute the findings.

\section{Abbreviation}

GDT, goal-directed therapy.

\section{Competing interests}

RMP has received honoraria from Edwards Lifesciences (Irvine, CA, USA), Pulsion Medical Systems (Munich, Germany), and Covidien Inc. (Dublin, Ireland) and research grants from LiDCO Ltd (Cambridge, UK) and USCOM Ltd (Sydney, Australia). NM declares that he has no competing interests.

\section{Authors' contributions}

Both authors read and approved the final manuscript.

\section{Acknowledgments}

RMP is a National Institute for Health Research (UK) Clinician Scientist.

Published: 3 June 2011

\section{References}

1. Cecconi M, Fasano N, Divella M, Gabriella Costa M, Rhodes A, Della Rocca G: Goal directed haemodynamic therapy during elective total hip arthroplasty under regional anaesthesia. Crit Care 2011, 15:R132

2. Jhanji S, Thomas B, Ely A, Watson D, Hinds CJ, Pearse RM: Mortality and utilization of critical care resources amongst high risk surgical patients in a large NHS Trust. Anaesthesia 2008, 63:695-700.

3. Pearse RM, Harrison D, James P, Watson D, Hinds CJ, Rhodes A, Grounds RM, Bennett ED: Identification and characterization of the high risk surgical population in the United Kingdom. Crit Care 2006, 10:R81.

4. Boyd O, Grounds RM, Bennett ED: A randomized clinical trial of the effect of deliberate perioperative increase of oxygen delivery on mortality in highrisk surgical patients. JAMA 1993, 270:2699-2707.

5. Wilson J, Woods I, Fawcett J, Whall R, Dibb W, Morris C, McManus E: Reducing the risk of major elective surgery: randomised controlled trial of preoperative optimisation of oxygen delivery. BMJ 1999, 318:1099-1103.

6. Pearse RM, Dawson D, Fawcett J, Rhodes A, Grounds RM, Bennett ED: Early goal-directed therapy after major surgery reduces complications and duration of hospital stay. A randomised, controlled trial. Crit Care 2005, 9:R687-R693.

7. Jhanji S, Vivian-Smith A, Lucena-Amaro S, Watson D, Hinds CJ, Pearse RM: Haemodynamic optimisation improves tissue microvascular flow and oxygenation after major surgery: a randomised controlled trial. Crit Care 2010, 14:R151

8. Sinclair $\mathrm{S}$, James $\mathrm{S}$, Singer $\mathrm{M}$ : Intraoperative intravascular volume optimisation and length of hospital stay after repair of proximal femoral fracture: randomised controlled trial. BMJ 1997, 315:909-912.

9. Venn R, Steele A, Richardson P, Poloniecki J, Grounds M, Newman P: Randomized controlled trial to investigate influence of the fluid challenge on duration of hospital stay and perioperative morbidity in patients with hip fractures. Br J Anaesth 2002, 88:65-71.

10. Steinbrook R: How best to ventilate? Trial design and patient safety in studies of the acute respiratory distress syndrome. N Eng/ J Med 2003, 348:1393-1401.

11. Hayes MA, Timmins AC, Yau EH, Palazzo M, Hinds CJ, Watson D: Elevation of systemic oxygen delivery in the treatment of critically ill patients. NEngl J Med 1994, 330:1717-1722.

\section{doi:10.1186/cc10011}

Cite this article as: MacDonald N, Pearse RM: Peri-operative hemodynamic therapy: only large clinical trials can resolve our uncertainty. Critical Care 2011, 15:122. 